\title{
A PARAMETRIZATION OF ISOMETRIC IMMERSIONS BETWEEN ANTI-DE SITTER SPACE-TIMES
}

\author{
Dedicated to Professors Haruo Kitahara and Syûkichi Tanno \\ on their sixtieth birthday
}

Kinetsu Abe ANd Hiroshi Mori

\begin{abstract}
The space of isometric immersions of an $n$-dimensional anti-de Sitter spacetime into another with codimension one is described in terms of certain families of countable $n$-tuples of real-valued functions.
\end{abstract}

\section{Introduction}

A fundamental problem in differential geometry is to characterize and determine all the submanifolds in a space form. A complete solution to the problem in the generality as stated above simply seems beyond the reach of the current mathematics. Historically, various conditions were imposed upon so as to make the problem somewhat more feasible, if not more viable. One of such conditions is to restrict submanifolds to being of codimension one and of the same constant curvature as the ambient space. The problem has received considerable attention under this rather restricted state; indeed, it has seen much progress.

For example, the problem has long been settled for the Riemannian space forms of non-negative curvature (see [5] and [11] for more information). In the hyperbolic case, only some partial solutions (see [2], [7], [9], [10]) existed until a lengthy but more complete description of the space was recently obtained (see [4]). In the indefinite case, Graves ([6]) gave the answer to the problem for the flat Lorentzian space forms. The case involving the de Sitter space forms was treated in [1].

Key words and phrases. isometric immersion, anti-de Sitter space-time, fundamental theorem for hypersurfaces, universal pseudo-Riemannian covering manifold.

Partly supported by the Grants-in-Aid for Scientific Research, the Ministry of Education, Science and Culture, Japan.

1991 Mathematics Subject Classification. Primary 53 C 42, Secondary 53 C 21.

Received January 9, 1997; revised October 7, 1997. 
In this paper, we take up the anti-de Sitter space forms of constant curvature -1 . We give a complete description of the space of the isometric immersions of $\widetilde{H}_{1}^{n}$ into $\widetilde{H}_{1}^{n+1}$. Here we denote by $\widetilde{H}_{1}^{n}$ the universal pseudo-Riemannian covering manifold of the $n$-dimensional anti-de Sitter space-time $H_{1}^{n}$.

Our main result, in essence, states: For each isometric immersion $\tilde{f}$ : $\widetilde{H}_{1}^{n} \rightarrow \widetilde{H}_{1}^{n+1}$, there exists a family of properly chosen, countable $n$-tuples of realvalued $C^{\infty}$ functions which completely describe the second fundamental form of the immersion; therefore, the immersion itself.

Conversely, given a family of properly chosen, countable n-tuples of real-valued $C^{\infty}$ functions defined over a family of mutually disjoint open intervals, there is an isometric immersion $\tilde{f}: \widetilde{H}_{1}^{n} \rightarrow \widetilde{H}_{1}^{n+1}$ whose relative nullity foliation is completely determined by functions in the family.

Throughout this paper, we make extensive use of the notion of relative nullity foliations. They are ideally suited for this study of submanifolds in the setting of this paper.

In Section 2, we briefly recall the universal pseudo-Riemannian manifold $\widetilde{H}_{1}^{n}$ of an $n$-dimensional anti-de Sitter space-time and show that no leaf of the relative nullity foliation associated with the isometric immersion $f: H_{1}^{n} \rightarrow H_{1}^{n+1}$ is a Lorentzian manifold with the induced metric.

In Section 3, we give a complete description of the relative nullity foliation of $f$. Central here is to make a proper choice for a family of $C^{\infty}$-functions defined in open intervals according to degeneracy or non-degeneracy of the leaves of the relative nullity foliation.

Section 4 is devoted to a precise statement of our main result and its proof.

\section{Preliminaries}

First, we recall the $n$-dimensional anti-de Sitter space-time $H_{1}^{n}$ (see [1] and [12] for details):

$$
H_{1}^{n}:=\left\{\left(y_{1}, y_{2}, \ldots, y_{n+1}\right) \in R_{2}^{n+1} ;-y_{1}^{2}-y_{2}^{2}+\sum_{i=3}^{n+1} y_{\imath}^{2}=-1\right\},
$$

with the indefinite metric $\langle\cdot, \cdot\rangle=-d y_{1}^{2}-d y_{2}^{2}+\sum_{l=3}^{n+1} d y_{\imath}^{2}$.

Next, we will define the universal pseudo-Riemannian covering manifold $\tilde{H}_{1}^{n}$ of $H_{1}^{n}$. We consider a mapping $\pi: R^{n} \rightarrow H_{1}^{n}$ given by

$$
\pi\left(x_{1}, x_{2}, \ldots, x_{n}\right)=\left(\sigma^{1 / 2} \cos x_{1}, \sigma^{1 / 2} \sin x_{1}, x_{2}, \ldots, x_{n}\right),
$$

$x_{1}, x_{2}, \ldots, x_{n} \in R$, where $\sigma=1+\sum_{\imath=2}^{n} x_{\imath}^{2}$. It is easy to show that the mapping $\pi$ is a covering mapping in the sense that for $x_{1}^{\prime}, \ldots, x_{n}^{\prime}$ and $x_{1}, \ldots, x_{n} \in R$, $\pi\left(x_{1}^{\prime}, x_{2}^{\prime}, \ldots, x_{n}^{\prime}\right)=\pi\left(x_{1}, x_{2}, \ldots, x_{n}\right)$ if and only if $x_{1}^{\prime}-x_{1}=2 \pi k,(k:$ integer $), x_{2}^{\prime}=$ $x_{\imath}, i=2,3, \ldots, n$. We also find that 


$$
\tilde{g}=\pi^{*}(\langle\cdot, \cdot\rangle)=-\sigma d x_{1}^{2}+\sum_{\imath, j=2}^{n}\left(\delta_{i j}-x_{\imath} x_{j} \sigma^{-1}\right) d x_{i} d x_{\jmath},
$$

where $\sigma=1+\sum_{\imath=2}^{n} x_{\imath}^{2}$. From this observation we can define a universal pseudoRiemannian covering manifold $\widetilde{H}_{1}^{n}$ of $H_{1}^{n}$ by

$$
\tilde{H}_{1}^{n}=\left(R^{n}, \tilde{g}\right) \text {. }
$$

We will begin with the following Lemma.

LEMmA 2.1. Suppose that $s \in(0,2 \pi), s \neq \pi,\left(a_{\imath \jmath}\right)_{3 \leqq \imath, \jmath \S n+1} \in S O(n-1)$ and $0<\alpha$, $\beta<\infty$ are given. Then we can find real numbers $t_{4}, \ldots, t_{n_{+}}$and $\phi, \theta \in[0,2 \pi)$ satisfying the following condition.

$$
\left\{\begin{array}{l}
\tau \cos \phi=\cos \theta \cos s-\cosh \alpha \sin \theta \sin s, \\
\tau \cosh \beta \sin \phi=\cos \theta \sin s+\cosh \alpha \sin \theta \cos s, \\
\tau \sinh \beta \sin \phi=a_{33} \sinh \alpha \sin \theta, \\
t_{\jmath}=a_{3 j} \sinh \alpha \sin \theta, \quad j=4, \ldots, n+1,
\end{array}\right.
$$

where $\tau=\left(1+\sum_{\imath=4}^{n+1} t_{\imath}^{2}\right)^{1 / 2}$.

Proof. First, we define real numbers $P$ and $Q$ by

$$
\left\{\begin{array}{l}
P=\left\{\left(a_{33} \lambda \cos s-\mu\right)^{2}+\left(a_{33} \sin s \sinh \alpha\right)^{2}\right\}^{1 / 2}, \\
Q=\left\{\left(a_{33} \lambda-\mu \cos s\right)^{2}+(\sin s \sinh \beta)^{2}\right\}^{1 / 2},
\end{array}\right.
$$

where $\lambda=\sinh \alpha \cosh \beta$ and $\mu=\cosh \alpha \sinh \beta$. Next, we define real numbers $t_{\imath}$ by

$$
t_{\imath}=Q^{-1} a_{3 i} \sinh \alpha \sinh \beta \sin s, \quad \imath=4, \ldots, n+1 .
$$

Note that for each $s \in(0,2 \pi)-\{\pi\}$ and each $\alpha, \beta \in(0, \infty)$, we have $Q>0$ and

$$
\tau=\left(1+\sum_{\imath=4}^{n+1} t_{\imath}^{2}\right)^{1 / 2}=P / Q
$$

Finally, we define $\phi, \theta \in[0,2 \pi)$ by

$$
\left\{\begin{array}{l}
\sin \phi=P^{-1} a_{33} \sin s \sinh \alpha, \\
\cos \phi=P^{-1}\left(a_{33} \cos s \sinh \alpha \cosh \beta-\cosh \alpha \sinh \beta\right), \\
\sin \theta=Q^{-1} \sin s \sinh \beta, \\
\cos \theta=Q^{-1}\left(a_{33} \sinh \alpha \cosh \beta-\cos s \cosh \alpha \sinh \beta\right) .
\end{array}\right.
$$

Using (2.5)-(2.8) we find that (2.4) is valid.

This completes the proof.

Lemma 2.1 implies the following. 
Proposition 2.1. No leaf of the relative nullity foliation associated to $f$ : $H_{1}^{n} \rightarrow H_{1}^{n+1}$ is a Lorentzian manifold with the induced metric.

Proof. Recall that any complete totally geodesic submanifold of codimension one intersects the center circle $S^{1}:=\left\{\left(x_{1}, x_{2}, 0, \ldots, 0\right) \in H_{1}^{n}\right\}$ once and only once in the projective sense. Thus any complete totally geodesic foliation of codimension one of a connected component $V$ of $U$ in $H_{1}^{n}$ is parametrized as follow.

Let $c(s)=(\cos s, \sin s, 0, \ldots, 0), a<s<b$, be the intersection of $V$ and the center circle $S^{1}$. For each $s \in(a, b)$ we denote by $L(s)$ the leaf of $\mathscr{F}$ passing through the point $c(s)$. Assume that for each $s \in(a, b), T_{c(s)} L(s)$, the tangent space of the $L(s)$ at the point $c(s)$ is a Lorentzian manifold. Then it is the intersection of $H_{1}^{n}$ and the vector subspace of $R_{2}^{n+1}$ which is generated by the vectors $c(s),\left(0,0, a_{i 3}(s), \ldots, a_{\imath n+1}(s)\right), i=4, \ldots, n+1$ and $\cosh \alpha(s) c^{\prime}(s)+\sinh \alpha(s)$ $\left(0,0, a_{33}(s), \ldots, a_{3 n+1}(s)\right)$, where $\alpha(s)$ and $a_{\imath j}(s), 3 \leqq \imath, j \leqq n+1$, are $C^{\infty}$ functions in $s$ and $\left(a_{\imath j}(s)\right)$ is in $S O(n-1)$. From this fact the foliation $\mathscr{F}$ on $V$ is parametrized as follows.

$$
\begin{gathered}
f:(a, b) \times S^{1} \times R^{n-2} \longrightarrow H_{1}^{n} \\
f\left(s, \theta, t_{4}, \ldots, t_{n+1}\right)=\lambda \cos \theta c(s)+\lambda \sin \theta\left\{\cosh \alpha(s) c^{\prime}(s)\right. \\
\left.+\sinh \alpha(s) e_{3}(s)\right\}+\sum_{i=4}^{n+1} t_{i} e_{i}(s),
\end{gathered}
$$

where $\lambda=\left(1+\sum_{\imath=4}^{n+1} t_{2}^{2}\right)^{1 / 2}, e_{i}(s)=\left(0,0, a_{i 3}(s), \ldots, a_{\imath n+1}(s)\right), i=3,4, \ldots, n+1$. We may, up to an isometry of $H_{1}^{n}$, assume that $a<0<b$ and $e_{i}(0)=(0, \ldots, 0,1,0$, $\ldots, 0),(1: i$ th coordinate), $i=3, \ldots, n+1$. Let $s \in(a, b), s \neq \pi$ fix. It follows from Lemma 1.1 there exist real numbers $t_{4}, \ldots, t_{n+1}, \phi, \theta$ such that

$$
f\left(0, \phi, t_{4}, \ldots, t_{n+1}\right)=f(s, \theta, 0, \ldots, 0) \text {. }
$$

In fact, setting $\beta=\alpha(0), \alpha=\alpha(s)$ we see that (2.9) is equivalent to the system of the equations in (2.4). From this observation we find that if the leaves of $\mathscr{F}$ are Lorentzian manifolds, then any two of them do intersect. This contradicts the definition of the foliation.

This completes the proof.

For later purpose we will introduce some notations and symbols. Let $\pi$ : $\widetilde{H}_{1}^{n} \rightarrow H_{1}^{n}$ (resp. $\pi^{\prime}: \widetilde{H}_{1}^{n+1} \rightarrow H_{1}^{n+1}$ ) be the universal pseudo-Riemannian covering mapping of the $n$-dimensional (resp. $(n+1)$-dimensional) anti-de Sitter space-time $H_{1}^{n}$ (resp. $H_{1}^{n+1}$ ) as in (2.1). Since the both mapping $\pi$ and $\pi^{\prime}$ are local isometries we can show that an isometric immersion $f: H_{1}^{n} \rightarrow H_{1}^{n+1}$ is lifted to an isometric immersion $\tilde{f}: \widetilde{H}_{1}^{n} \rightarrow \widetilde{H}_{1}^{n+1}$, that is, $\pi^{\prime} \circ \tilde{f}=f \circ \pi: \widetilde{H}_{1}^{n} \rightarrow H_{1}^{n+1}$.

Let $\tilde{f}: \widetilde{H}_{1}^{n} \rightarrow \widetilde{H}_{1}^{n+1}$ be an isometric immersion. Denote by $\tilde{A}$ the shape operator of $\tilde{f}$ relative to a unique (up to a sign) field of unit normals along $\hat{f}$. Recall that $\widetilde{H}_{1}^{n}=\left(R^{n}, \tilde{g}\right)$. Let $\tilde{a}_{\imath}$ be the components of $\tilde{A}$ with respect to the 
standard coordinates $\left\{x_{1}, \ldots, x_{n}\right\}$ of $R^{n}$

$$
\tilde{A}\left(\frac{\partial}{\partial x_{\imath}}\right)=\sum_{j=1}^{n} \tilde{a}_{\imath j} \frac{\partial}{\partial x_{j}} \text {. }
$$

We will, for simplicity, say that the $\tilde{a}_{\imath}$ are components for $\tilde{f}$.

\section{Description of foliation}

Let $f: H_{1}^{n} \rightarrow H_{1}^{n+1}$ be an isometric immersion. Denote by $\mathscr{F}$ the relative nullity foliation on $H_{1}^{n}$ determined by $f$. We know (see [3]) that $\mathscr{F}$ is a codimension one complete totally geodesic foliation in an open subset $U$ of $H_{1}^{n}$. By $\mathscr{I} \mid U_{\alpha}$ we mean the restriction of $\mathscr{F}$ to a connected component $U_{\alpha}$ of $U$. From Lemma 3 in [1] and Proposition 2.1 in this paper we find that the leaves of $\mathscr{F} \mid U_{\alpha}$ are either all degenerate submanifolds of $H_{1}^{n}$ or all Riemannian submanifolds of $H_{1}^{n}$. Note that the center circle $S^{1}$ in $H_{1}^{n}$ is parametrized by

$$
c(s)=(\cos s, \sin s, 0, \ldots, 0), \quad-\pi<s \leqq \pi .
$$

We find that each leaf $L$ of $\mathscr{F} \mid U_{\alpha}$ meets the center circle at a unique point. In fact, since $L$ is a connected, complete totally geodesic hypersurface of $H_{1}^{n}$, it is a connected component of the intersection of $V \cap H_{1}^{n}$, where $V$ is a linear hyperplane in $R_{2}^{n+2}$ which meets $H_{1}^{n}$ (see [12], pp. 62-68). From this observation and the very definition of foliation it follows that $V$ meets $S^{1}$ at one point, but cannot contain the center circle.

3.1. Foliation with degenerate leaves. We will first consider the case where leaves of $\mathscr{F} \mid U_{\alpha}$ are degenerate submanifolds of $H_{1}^{n}$. We know (see [1]) that along the center circle $c(s)=(\cos s, \sin s, 0, \ldots, 0)$ in $H_{1}^{n}$, there exists a parallel field $e(s)$ (resp. $V(s)$ ) of null vectors (resp. $(n-2)$-dimensional Riemannian vector spaces) tangent to the leaves of $\mathscr{F}$. Note that $e(s)$ may be written in the form

$$
e(s)=\left(-\cos s, \sin s, b_{2}(s), \ldots, b_{n}(s)\right), \quad a<s<b,
$$

where $b_{2}(s), \ldots, b_{n}(s)$ are $C^{\infty}$ functions defined in the open interval $(a, b)$ satisfying the condition $\sum_{\imath=2}^{n} b_{i}^{\prime}(s)^{2} \equiv 1$. Since $e(s)$ is parallel along the curve $c(s)$, the Weingarten formula implies that all the functions $b_{i}(s)$ are constant. From this fact together with that $V(s)$ is also parallel, we find that $V(s)$ is a constant $(n-2)$-dimensional vector space in $R^{n-1}$. Here and in what follows, we will canonically identify the $(n-1)$-dimensional Euclidean space $R^{n-1}$ with the subset $\{(0,0)\} \times R^{n-1}$ of $R_{2}^{n+1}$. From this observation we have the following description of the relative nullity foliation whose leaves are degenerate.

PROPOSITION 3.1. Suppose that the leaves of $\mathscr{I} \mid U_{\alpha}$ are all degenerate manifolds. Then, the open subset $U_{\alpha}$ is parametrized by a mapping $\phi:(a, b) \times R^{n-1}$ 
$\rightarrow H_{1}^{n}$

$$
\phi\left(s, t_{2}, t_{3}, \ldots, t_{n}\right)=\tau c(s)+t_{2} c^{\prime}(s)+\sum_{\imath=2}^{n} t_{i} e_{\imath},
$$

$s \in(a, b)$, and $t_{2}, \ldots, t_{n} \in R$, where $\tau=\left(1+\sum_{\imath=3}^{n} t_{2}^{2}\right)^{1 / 2}$. The second fundamental form $\Pi$ of $f \mid U_{\alpha}$, at the point $\phi\left(s, t_{2}, \ldots, t_{n}\right)$, is given by

$$
\Pi=r(s) \tau d s^{2},
$$

where $r(s)$ is $a C^{\infty}$ function defined in the open interval $(a, b)$.

Proof. First, note that the mapping $\phi$ is an imbedding. Setting $t_{1}=s$ and denoting by $g_{\imath}$, the components of the induced indefinite Riemannian metric $\phi^{*}(\langle\cdot, \cdot\rangle)$ we have that

$$
\left\{\begin{array}{l}
g_{11}=-\left(t_{2}^{2}+\tau^{2}\right), \\
g_{12}=-\tau, \\
g_{1 \imath}=t_{2} t_{\imath} \tau^{-1}, \quad i=3, \ldots, n, \\
g_{2 i}=0, \quad i=2,3, \ldots, n, \\
g_{\imath \jmath}=\delta_{i j}-t_{i} t_{j} \tau^{-2}, \quad i, j=3, \ldots, n,
\end{array}\right.
$$

where $\delta_{i j}$ is the Kronecker's delta. Denote by $g^{i j}$ the components of the inverse matrix of the matrix $\left(g_{\imath \jmath}\right)$. Then, from (3.3) it follows that

$$
\begin{cases}g^{1 \imath}=-\delta_{2 i} \tau^{-1}, & i=1,2, \ldots, n, \\ g^{i \jmath}=\delta_{i j}+t_{i} t_{\jmath}, & i, j=2, \ldots, n .\end{cases}
$$

Let $A$ be the shape operator of the isometric immersion $f$ relative to a unique (up to a sign) field of unit normals along $f$. Recall that for each fixed $s \in(a, b)$, the mapping $\left(t_{2}, \ldots, t_{n}\right) \in R^{n-1} \mapsto \phi\left(s, t_{2}, \ldots, t_{n}\right) \in H_{1}^{n}$ defines a leaf of the foliation $\mathscr{F} \mid U_{\alpha}$. From this observation it follows that

$$
\left\{\begin{array}{l}
A\left(\phi_{*} \frac{\partial}{\partial s}\right)=r \phi_{*} \frac{\partial}{\partial t_{2}}, \\
A\left(\phi_{*} \frac{\partial}{\partial t_{\imath}}\right)=0, \quad i=2, \ldots, n,
\end{array}\right.
$$

where $r$ is a function of $s, t, t_{3}, \ldots, t_{n}$.

From the Codazzi equations together with (3.3), (3.4) and (3.5) it follows that the function $r$ depends only on $s$.

This complete the proof.

We will consider an isometric immersion $\tilde{f}: \widetilde{H}_{1}^{n} \rightarrow \widetilde{H}_{1}^{n+1}$, which covers the restriction $f \mid \phi\left((a, b) \times R^{n-1}\right)$, and calculate the coefficients $\tilde{a}_{\imath \jmath}$ of the second 
fundamental form of $\tilde{f}$. Using (2.1) and (3.2) we can naturally lift the mapping $\phi$ to a mapping $\tilde{\phi}:(a, b) \times R^{n-1} \rightarrow \widetilde{H}_{1}^{n}=\left(R^{n}, \tilde{g}\right)$ in the form.

$$
\tilde{\phi}\left(s, t_{2}, \ldots, t_{n}\right)=\left(s+\tan ^{-1}\left(t_{2} \tau^{-1}\right), 0, \ldots, 0\right)+\sum_{i=2}^{n} t_{i} \tilde{e}_{\imath}
$$

where $\tau=\left(1+\sum_{\imath=3}^{n} t_{\imath}^{2}\right)^{1 / 2}$ and $\tilde{e}_{\imath}:=\left(0, b_{i 2}, \ldots, b_{i n}\right), i=2,3, \ldots, n$. Define the vector fields $X_{\imath}$ on $(a, b) \times R^{n-1}$ by

$$
\left\{\begin{array}{l}
X_{1}=\frac{\partial}{\partial s} \\
X_{\imath}=-R_{\imath} \tau^{-1} T^{-2} \frac{\partial}{\partial s}+\sum_{j=2}^{n} b_{j i} \frac{\partial}{\partial t_{j}}, \quad i=2, \ldots, n,
\end{array}\right.
$$

where $\tau=\left(1+\sum_{\imath=3}^{n} t_{\imath}^{2}\right)^{1 / 2}, T=\left(t_{2}^{2}+\tau^{2}\right)^{1 / 2}$ and $R_{\imath}=T^{2} b_{2 i}-\sum_{j=2}^{n} t_{2} t_{j} b_{j i}$. Then from (3.6) and (3.7) it follows that

$$
\left(\frac{\partial}{\partial x_{\imath}}\right)_{\tilde{\phi}}=\tilde{\phi}_{*} X_{\imath}, \quad i=1,2, \ldots, n .
$$

From the fact that $\pi$ (resp. $\pi^{\prime}$ ) is a local isometry of $\widetilde{H}_{1}^{n}$ onto $H_{1}^{n}$ (resp. $\widetilde{H}_{1}^{n+1}$ onto $H_{1}^{n+1}$ ) together with $(2.10),(3.2)$ and (3.6) we find that the values of $\tilde{a}_{\imath \jmath}$, at the point $\tilde{\phi}\left(s, t_{2}, \ldots, t_{n}\right)$, are given by

$$
\left\{\begin{array}{l}
\tilde{a}_{11}=r(s) \tau T^{-2}, \\
\tilde{a}_{1 \jmath}=r(s) b_{2 \jmath}, \\
\tilde{a}_{i 1}=-\tilde{a}_{11} R_{i} \tau^{-1} T^{-2}, \\
\tilde{a}_{\imath \jmath}=-\tilde{a}_{1 j} R_{i} \tau^{-1} T^{-2},
\end{array}\right.
$$

$i, j=2, \ldots, n$, where $\tau, T$ and $R_{\imath}$ are given as above. Recall (see [8], p. 24) that for any $C^{\infty}$ function $h$ defined in the open subset $\Omega:=\tilde{\phi}\left((a, b) \times R^{n-1}\right)$ of $\widetilde{H}_{1}^{n}$ we have that

$$
\frac{\partial}{\partial x_{j}}(h)=\left\{\frac{\partial}{\partial t_{j}}(h \circ \tilde{\phi})\right\} \circ \tilde{\phi}^{-1},
$$

$j=1, \ldots, n$. Nate that the composite functions $\tilde{a}_{\imath j^{\circ}} \tilde{\phi}$ are $C^{\infty}$ in $(a, b) \times R^{n-1}$, and are analytic functions of the variables $t_{2}, \ldots, t_{n}$. From this observation it follows that the coefficients of the second fundamental form are $C^{\infty}$ in a neighborhood of the closure of the subset $\tilde{\phi}\left((a, b) \times R^{n-1}\right)$ in $\widetilde{H}_{1}^{n}$ if and only if we have that

$$
\frac{d^{\jmath}}{d s^{\jmath}} r(s) \longrightarrow 0, \quad j=0,1,2, \ldots,
$$

as $s$ approaches the end points of $(a, b)$.

3.2. Foliation with Riemannian leaves. We will next consider the case 
where leaves of $\mathscr{I} \mid U_{\alpha}$ are Riemannian submanifolds of $H_{1}^{n}$. Along the intersection $U_{\alpha} \cap S^{1}$ we can find a $C^{\infty}$ function $\theta(s)$ and a $C^{\infty}$ field of orthonormal vectors $e_{2}(s), \ldots, e_{n}(s)$ of $R^{n-1}$ to describe the foliation $\mathscr{F} \mid U_{\alpha}$ as follows. As in the previous subsection, we will canonically identify the $(n-1)$-dimensional Euclidean space $R^{n-1}$ with the subset $\{(0,0)\} \times R^{n-1}$ of $R_{2}^{n+1}$.

On the function $\theta(s)$ and the fields of orthonormal vectors $e_{2}(s), \ldots, e_{n}(s)$ we will impose the conditions (3.11), (3.13) and (3.14) below which are necessary and sufficient conditions for that the parametrization $\psi$ of (3.15) below represent the foliation $\mathscr{F} \mid U_{\alpha}$.

We will, first, write down the preliminary conditions.

$$
\left\langle e_{i}^{\prime}(s), e_{j}(s)\right\rangle=0, \quad \text { for } 3 \leqq i, j \leqq n,
$$

where $\langle$,$\rangle is the Euclidean inner product of R^{n-1}$. We can easily shown that operating a suitable $C^{\infty} S O(n-2)$-valued function defined in the open interval $(a, b),(3.11)$ is always satisfied. Let $k_{3}(s), \ldots, k_{n}(s)$ be $C^{\infty}$ functions uniquely determined in the open interval $(a, b)$, subject to the following conditions.

$$
\left\{\begin{array}{l}
e_{2}^{\prime}(s)=\sum_{i=3}^{n} k_{i}(s) e_{i}(s), \\
e_{i}^{\prime}(s)=-k_{i}(s) e_{2}(s), \quad i=3, \ldots, n .
\end{array}\right.
$$

Conditions we impose are as follows.

$$
\begin{gathered}
\theta^{\prime}(s)^{2}+\sum_{i=3}^{n} k_{i}(s)^{2} \sinh ^{2} \theta(s) \leqq \cosh ^{2} \theta(s), \\
\left\{\begin{array}{l}
\left|\theta^{\prime}(s)\right|<\cosh \theta(s), \text { when } \cosh \theta(s) \leqq \sqrt{2}, \\
\left|\theta^{\prime}(s)\right|<2|\tanh \theta(s)|, \text { when } \sqrt{2}<\cosh \theta(s) .
\end{array}\right.
\end{gathered}
$$

PROPOSITION 3.2. Suppose that the leaves of $\mathscr{F} \mid U_{\alpha}$ are all Riemannian manifolds. Then, the open subset $U_{\alpha}$ is parametrized by a mapping $\phi:(a, b) \times R^{n-1} \rightarrow$ $H_{1}^{n}$ defined by

$$
\begin{aligned}
\psi\left(s, t_{2}, t_{3}, \ldots, t_{n}\right)= & \left(1+\sum_{i=2}^{n} t_{2}^{2}\right)^{1 / 2} c(s)+t_{2} \sinh \theta(s) c^{\prime}(s) \\
& +t_{2} \cosh \theta(s) e_{2}(s)+\sum_{i=3}^{n} t_{i} e_{i}(s),
\end{aligned}
$$

$s \in(a, b)$ and $t_{2}, \ldots, t_{n} \in R$. The condition (3.13) is a necessary and sufficient condition for the mapping $\phi$ to be an immersion. The conditions (3.14) is a necessary and sufficient condition that for each fixed $s \in(a, b)$, the mapping $\left(t_{2}, \ldots\right.$, $\left.t_{n}\right) \mapsto \phi\left(s, t_{2}, \ldots, t_{n}\right)$ represents a leaf $L(s)$ of the foliation $\mathscr{F} \mid U_{\alpha}$. The second fundamental form $I I$ of $f \mid U_{\alpha}$, at the point $\phi\left(s, t_{2}, \ldots, t_{n}\right)$, is given by

$$
\Pi=r(s)\left(T \cosh \theta(s)+t_{2} \theta^{\prime}(s)+W \sinh \theta(s)\right) d s^{2},
$$


where $T=\left(1+\sum_{\imath=2}^{n} t_{\imath}^{2}\right)^{1 / 2}, W=\sum_{\jmath=3}^{n} t_{j} k_{j}(s)$ and $r(s)$ is a $C^{\infty}$ function defined in the open interval $(a, b)$.

Proof. Setting $t_{1}=s$ and denoting by $g_{\imath}$, by the components of the induced indefinite Riemannian metric $\psi^{*}(\langle\cdot, \cdot\rangle)$ we have the following.

$$
\left\{\begin{aligned}
g_{11}= & -t_{2}^{2}\left(\sinh ^{2} \theta(s)+\theta^{\prime}(s)^{2}\right)-T^{2}-2 t_{2} \theta^{\prime}(s) \cosh \theta(s) T \\
& -2 t_{2} \theta^{\prime}(s) \sinh \theta(s) W+W^{2}+\sum_{j=3}^{n}\left(t_{2} k_{j}(s) \cosh \theta(s)\right)^{2}, \\
g_{12}= & -\left(T-t_{2}^{2} T^{-1}\right) \sinh \theta(s)-\cosh \theta(s) W, \\
g_{1 \jmath}= & t_{2} t_{j} T^{-1} \sinh \theta(s)+t_{2} k_{j}(s) \cosh \theta(s), \quad 3 \leqq j \leqq n, \\
g_{\imath \jmath}= & \delta_{i j}-t_{i} t_{j} T^{-2}, \quad 2 \leqq i, j \leqq n .
\end{aligned}\right.
$$

Denote by $\Delta$ by the determinant of the matrix $\left(g_{\imath j}\right)$. Using the Laplace expansion theorem for $\Delta$ we find that

$$
\Delta=-T^{-2}\left(T \cosh \theta(s)+W \sinh \theta(s)+t_{2} \theta^{\prime}(s)\right)^{2} .
$$

Note that $q:=T \cosh \theta(s)+t_{2} \theta^{\prime}(s)+W \sinh \theta(s)$ is positive when $t_{2}, \ldots, t_{n}=0$. Using Schwartz inequality we have that for all $t_{2}, \ldots, t_{n} \in R$,

$$
q \geqq T \cosh \theta(s)-\left\{\left(T^{2}-1\right)\left(\theta^{\prime}(s)^{2}+\sum_{i=3}^{n} k_{i}(s)^{2} \sinh ^{2} \theta(s)\right)\right\}^{1 / 2},
$$

with equality $\theta^{\prime}(s)=\lambda t_{2}, k_{i}(s) \sinh \theta(s)=\lambda t_{\imath}, i=3, \ldots, n$ for some constant $\lambda \leqq 0$. From this observation it follows that the condition (3.13) is a necessary and sufficient condition that the mapping $\phi$ be an immersion.

Denote by $g^{i j}$ the components of the inverse matrix of the matrix $\left(g_{i j}\right)$. Using the Laplace expansion theorem for $\Delta$ we find that

$$
\left\{\begin{array}{l}
g^{11}=-\left(T \cosh \theta(s)+W \sinh \theta(s)+t_{2} \theta^{\prime}(s)\right)^{-2}, \\
g^{12}=g^{11}(T \sinh \theta(s)+\cosh \theta(s) W), \\
g^{12}=-g^{11}\left(t_{2} k_{i}(s) \cosh \theta(s)\right), \quad i=3,4, \ldots, n .
\end{array}\right.
$$

Defining $\tau=\left(T^{2}+t_{2}^{2} \sinh ^{2} \theta(s)\right)^{1 / 2}$ and $\tan ^{-1}\left(t_{2} T^{-1} \sinh \theta(s)\right)=\omega\left(s, t_{2}, \ldots, t_{n}\right)=: \omega$ we find that

$$
\begin{aligned}
\psi\left(s, t_{2}, \ldots, t_{n}\right)= & \tau(\cos (s+\omega), \sin (s+\omega), 0, \ldots, 0) \\
& +t_{2} \cosh \theta(s) e_{2}(s)+\sum_{i=3}^{n} t_{i} e_{i}(s),
\end{aligned}
$$

where $e_{i}(s)=\left(0,0, b_{i 2}(s), \ldots, b_{i n}(s)\right), i=2, \ldots, n$.

To prove the third assertion in Proposition 3.2 it is sufficient to show that (3.14) holds if and only if

$$
|\partial \omega / \partial s|=\left|\tau^{-2} T t_{2} \theta^{\prime}(s) \cosh \theta(s)\right|<1
$$


for all $t_{2}, \ldots, t_{n} \in R$ and for all $s \in(a, b)$. Putting $x=t_{2}^{2}, y=\sum_{\imath=3}^{n} t_{\imath}^{2}$ and $m=$ $\cosh \theta(s)$, we find that

$$
\partial \omega / \partial s=h(s, x, y) \theta^{\prime}(s)
$$

where

$$
h(s, x, y)=\frac{m \sqrt{(1+x+y) x}}{1+m^{2} x+y}, \quad 0 \leqq x, y<\infty .
$$

Since

$$
\partial h(s, x, y) / \partial x=\frac{(1+y) m\left\{\left(2-m^{2}\right) x+1+y\right\}}{2 \sqrt{x(1+x+y)\left(1+m^{2} x+y\right)}}
$$

for $0<x<\infty, 0 \leqq y<\infty$, we have that

$$
\left\{\begin{array}{c}
0 \leqq h(s, x, y)<h(s, \infty, y)=\cosh \theta(s), \\
\text { when } \cosh \theta(s) \leqq \sqrt{2} \\
0 \leqq h(s, x, y) \leqq h\left(s, \frac{1+y}{m^{2}-2}, y\right)=2|\operatorname{coth} \theta(s)|, \\
\text { when } \sqrt{2}<\cosh \theta(s) .
\end{array}\right.
$$

From this observation, we see that our assertion holds.

Denote by $A$ the shape operator of $f$ relative to a field of unit normals along $f$. From (3.16) and (3.17) it follows that

$$
A\left(\phi_{*} \frac{\partial}{\partial s}\right)=r\left(-g^{11}\right)^{-1 / 2} \sum_{j=1}^{n} g^{1 j} \psi_{*}\left(\frac{\partial}{\partial t_{\jmath}}\right),
$$

where $r$ is a function of $s, t_{2}, \ldots, t_{n}$. Denote by $\nabla_{\imath}$ the covariant differentiation with respect to the $i$-th coordinate vector field $\partial / \partial t_{\imath}$. Then, we get that

$$
\sum_{\jmath=1}^{n} \nabla_{i}\left(\left(-g^{11}\right)^{-1 / 2} g^{1 j} \psi_{*} \frac{\partial}{\partial t_{j}}\right)=0, \quad i=2, \ldots, n .
$$

In fact, from the definition of the Christoffel's symbols it follows that

$$
\begin{aligned}
\sum_{j=1}^{n} \nabla_{i}( & \left.\left(-g^{11}\right)^{-1 / 2} g^{1 \jmath} \psi_{*} \frac{\partial}{\partial t_{\jmath}}\right)=\frac{1}{2}\left(-g^{11}\right)^{-3 / 2} \sum_{l, h, \jmath=1}^{n} g^{1 h} \\
& \times\left\{g^{11} g^{j l}\left(\frac{\partial g_{l h}}{\partial t_{\imath}}-\frac{\partial g_{l \imath}}{\partial t_{h}}+\frac{\partial g_{i h}}{\partial t_{l}}\right)-g^{1 l} g^{1 \jmath} \frac{\partial g_{l h}}{\partial t_{\imath}}\right\} \psi_{*} \frac{\partial}{\partial t_{\jmath}} .
\end{aligned}
$$

Codazzi equations then imply that the function $r$ is a function of $s$ only.

This completes the proof.

As in the previous subsection, we consider an isometric immersion $\tilde{f}: \tilde{H}_{1}^{n} \rightarrow$ $\tilde{H}_{1}^{n+1}$, which covers the restriction $f \mid \phi\left((a, b) \times R^{n-1}\right)$, and calculate the coefficients $\tilde{a}_{\imath}$ of the second fundamental form of $\tilde{f}$. 
Using (2.1) and (3.15) we can naturally lift the mapping $\phi$ to a mapping $\tilde{\psi}:(a, b) \times R^{n-1} \rightarrow \widetilde{H}_{1}^{n}=\left(R^{n}, \tilde{g}\right)$ in the form.

$$
\begin{aligned}
\tilde{\phi}\left(s, t_{2}, \ldots, t_{n}\right)= & \left(s+\tan ^{-1}\left(t_{2} T^{-1} \sinh \theta(s)\right), 0, \ldots, 0\right) \\
& +t_{2} \cosh \theta(s) \tilde{e}_{2}(s)+\sum_{i=3}^{n} t_{i} \tilde{e}_{i}(s),
\end{aligned}
$$

where $T=\left(1+\sum_{\imath=2}^{n} t_{\imath}^{2}\right)^{1 / 2}, e_{i}(s)=\left(0, b_{i 2}(s), \ldots, b_{i n}(s)\right), i=2,3, \ldots, n$. We define the vector fields $X_{\imath}, i=1, \ldots, n$, in the product space $(a, b) \times R^{n-1}$ by

$$
\left\{\begin{array}{l}
X_{1}=\frac{T \cosh \theta(s)}{Q} X, \\
X_{\imath}=-\frac{R_{\imath} \sinh \theta(s)}{P Q} X+Y_{\imath}, \quad i=2, \ldots, n,
\end{array}\right.
$$

where $P=T^{2}+t_{2}^{2} \sinh ^{2} \theta(s), Q=T \cosh \theta(s)+t_{2} \theta^{\prime}(s)+\sigma \sinh \theta(s), \sigma=\sum_{j=3}^{n} t_{j} k_{j}(s)$ and $R_{\imath}=\left(T^{2}-t_{2}^{2}\right) b_{2 i}(s)-\sum_{j=3}^{n} t_{2} t_{j} b_{j i}(s) \cosh \theta(s)$. Here we define the vector fields $X$ and $Y_{\imath}$ by

$$
\left\{\begin{array}{l}
X=\frac{\partial}{\partial s}+\frac{-t_{2} \sinh \theta(s) \theta^{\prime}(s)+\sigma}{\cosh \theta(s)} \frac{\partial}{\partial t_{2}}-\sum_{j=3}^{n} t_{2} k_{j}(s) \cosh \theta(s) \frac{\partial}{\partial t_{j}}, \\
Y_{2}=\frac{b_{2 i}(s)}{\cosh \theta(s)} \frac{\partial}{\partial t_{2}}+\sum_{j=3}^{n} b_{j i}(s) \frac{\partial}{\partial t_{j}}, \quad i=2, \ldots, n .
\end{array}\right.
$$

From (3.15) and (3.23) it follows that

$$
\left(\frac{\partial}{\partial x_{\imath}}\right)_{\tilde{\phi}}=\tilde{\phi}_{*}\left(X_{\imath}\right), \quad i=1,2, \ldots, n .
$$

From (3.2), (3.15) and (3.24) together with that $\pi$ is a local isometry, we find that the values of $\tilde{a}_{\imath \jmath}$, at the point $\tilde{\psi}\left(s, t_{2}, \ldots, t_{n}\right)$, are given by

$$
\left\{\begin{array}{l}
\tilde{a}_{11}=-r(s) T^{2}(P Q)^{-1} \cosh ^{2} \theta(s), \\
\tilde{a}_{1 \jmath}=-r(s) T Q^{-1} \cosh \theta(s) \sinh \theta(s) b_{2 j}(s), \\
\tilde{a}_{i 1}=-\tilde{a}_{11} R_{i}(T P)^{-1} \tanh \theta(s), \\
\tilde{a}_{\imath \jmath}=-\tilde{a}_{1 j} R_{i}(T P)^{-1} \tanh \theta(s),
\end{array}\right.
$$

$i, j=2, \ldots, n$, where $P, Q, \sigma$ and $R_{\imath}$ are given as above. By similar arguments in the previous subsection, we can show that the coefficients of the second fundamental form are $C^{\infty}$ in a neighborhood of the closure of the subset $\tilde{\psi}((a, b) \times$ $\left.R^{n-1}\right)$ in $\widetilde{H}_{1}^{n}$ if and only if we have that as $s$ approaches the end points of $(a, b)$,

$$
X_{1}^{l_{1}} X_{2}^{l_{2}} \cdots X_{n}^{l_{n}}\left(\tilde{a}_{\imath j}\right) \longrightarrow 0
$$

for all nonnegative integers $l_{1}, l_{2}, \ldots, l_{n}$. 


\section{Proof of theorem}

Let $\tilde{f}: \widetilde{H}_{1}^{n} \rightarrow \widetilde{H}_{1}^{n+1}$ be an isometric immersion. Denote by $\tilde{\mathcal{F}}$ the relative nullity foliation on $\tilde{H}_{1}^{n}$ determined by $\tilde{f}$. By the same argument as in the previous section we can show (see [3]) that $\tilde{\mathcal{F}}$ is a codimension one complete totally geodesic foliation in an open subset $\tilde{U}$ of $\tilde{H}_{1}^{n}$. By $\tilde{\mathscr{I}} \mid \widetilde{U}_{\alpha}$ we mean the restriction of $\tilde{F}$ to a connected component $\tilde{U}_{\alpha}$ of $\tilde{U}$. We also find that the leaves of $\tilde{\mathscr{F}} \mid \tilde{U}_{\alpha}$ are either all degenerate submanifolds of $\widetilde{H}_{1}^{n}$ or all Riemannian submanifolds of $\widetilde{H}_{1}^{n}$.

We first consider the case where the leaves of $\tilde{\mathscr{F}} \mid \tilde{U}_{\alpha}$ are degenerate manifolds. In $\tilde{U}_{\alpha}$ we have a parametrization $\tilde{\phi}_{\alpha}:\left(a_{\alpha}, b_{\alpha}\right) \times R^{n-1} \rightarrow \widetilde{H}_{1}^{n}=\left(R^{n}, \tilde{g}\right)$ given by

$$
\tilde{\phi}_{\alpha}\left(s, t_{2}, \ldots, t_{n}\right)=\left(s+\tan ^{-1}\left(t_{2} \tau^{-1}\right), 0, \ldots, 0\right)+\sum_{j=2}^{n} t_{i} \tilde{e}_{2 \alpha},
$$

$a_{\alpha}<s<b_{\alpha},-\infty<t_{2}, \cdots, t_{n}<\infty$, where $\tau=\left(1+\sum_{\imath=3}^{n} t_{\imath}^{2}\right)^{1 / 2}$ and $\left\{\tilde{e}_{\imath \alpha}:=\left(0, b_{i 2 \alpha}, \ldots\right.\right.$, $\left.\left.b_{i n \alpha}\right), i=2,3, \ldots, n\right\}$ is an orthonormal basis of the Euclidean space $R^{n-1} \cong\{0\} \times$ $R^{n-1} \subset R^{n}$ along $\left(a_{\alpha}, b_{\alpha}\right)$. The components $\tilde{a}_{\imath \jmath}$, at the point $\tilde{\phi}_{\alpha}\left(s, t_{2}, \ldots, t_{n}\right)$, of the shape operator of the isometric immersion $\tilde{f}$, are given by

$$
\left\{\begin{array}{l}
\tilde{a}_{11}=r_{\alpha}(s) \tau T^{-2}, \\
\tilde{a}_{1 \jmath}=r_{\alpha}(s) b_{2 \jmath \alpha}, \\
\tilde{a}_{i 1}=-\tilde{a}_{11} R_{\imath \alpha} \tau^{-1} T^{-2}, \\
a_{\imath \jmath}=-\tilde{a}_{1 \jmath} R_{\imath \alpha} \tau^{-1} T^{-2},
\end{array}\right.
$$

$i, j=2, \ldots, n$, where $\tau=\left(1+\sum_{\imath=3}^{n} t_{\imath}^{2}\right)^{1 / 2}, T=\left(t_{2}^{2}+\tau^{2}\right)^{1 / 2}$ and $R_{\imath \alpha}=T^{2} b_{2 \imath \alpha}-\sum_{\jmath=2}^{n} t_{2} t_{j} b_{j i \alpha}$. The function $r_{\alpha}(s) \in C^{\infty}\left(a_{\alpha}, b_{\alpha}\right)$ satisfies

$$
\frac{d^{\jmath}}{d s^{\jmath}} r_{\alpha}(s) \longrightarrow 0, \quad j=0,1,2, \ldots,
$$

as $s$ approaches the end points of $\left(a_{\alpha}, b_{\alpha}\right)$.

We next consider the case where the leaves of $\tilde{\mathcal{F}} \mid \tilde{U}_{\beta}$ are Riemannian manifolds. In $\tilde{U}_{\beta}$ we have a parametrization $\tilde{\psi}_{\beta}:\left(a_{\beta}, b_{\beta}\right) \times R^{n-1} \rightarrow \widetilde{H}_{1}^{n}=\left(R^{n}, \tilde{g}\right)$ given by

$$
\begin{aligned}
\tilde{\psi}_{\beta}\left(s, t_{2}, \ldots, t_{n}\right)= & \left(s+\tan ^{-1}\left(t_{2} T^{-1} \sinh \theta_{\beta}(s)\right), 0, \ldots, 0\right) \\
& +t_{2} \cosh \theta_{\beta}(s) \tilde{e}_{2 \beta}(s)+\sum_{i=3}^{n} t_{i} \tilde{e}_{\imath \beta}(s),
\end{aligned}
$$

$a_{\beta}<s<b_{\beta},-\infty<t_{2}, \ldots, t_{n}<\infty$, where $T=\left(1+\sum_{i=2}^{n} t_{i}^{2}\right)^{1 / 2}$ and $\left\{\tilde{e}_{i \beta}(s)=\left(0, b_{i 2 \beta}(s)\right.\right.$, $\left.\left.\ldots, b_{i n \beta}(s)\right), i=2,3, \ldots, n\right\}$ is a $C^{\infty}$ field of orthonormal vectors of the Euclidean space $R^{n-1} \cong\{0\} \times R^{n-1} \subset R^{n}$ along $\left(a_{\beta}, b_{\beta}\right)$. Note that the field $\left\{\tilde{e}_{i \beta}(s), i=2, \ldots\right.$, $n\}$ is uniquely determined by $(n-2) C^{\infty}$ functions $k_{3 \beta}(s), \ldots, k_{n \beta}(s)$ defined in $\left(a_{\beta}, b_{\beta}\right)$. Defined by $\langle$,$\rangle is the Euclidean inner product of R^{n-1} \cong\{0\} \times R^{n-1}$. We assume that the functions $\theta_{\beta}(s)$ and $k_{\imath \beta}(s), i=3, \ldots, n$, satisfy the following 
conditions :

$$
\begin{gathered}
\left\langle\tilde{e}_{i \beta}^{\prime}(s), \tilde{e}_{j \beta}(s)\right\rangle=0, \quad \text { for } \quad 3 \leqq i, j \leqq n, \\
\left\{\begin{array}{l}
\tilde{e}_{2 \beta}^{\prime}(s)=\sum_{i=3}^{n} k_{i \beta}(s) \tilde{e}_{i \beta}(s), \\
\tilde{e}_{i \beta}^{\prime}(s)=-k_{\imath \beta}(s) \tilde{e}_{2 \beta}(s), \quad i=3, \ldots, n,
\end{array}\right. \\
\theta_{\beta}^{\prime}(s)^{2}+\sum_{i=3}^{n} k_{i \beta}(s)^{2} \sinh ^{2} \theta_{\beta}(s) \leqq \cosh ^{2} \theta_{\beta}(s), \\
\left\{\begin{array}{l}
\left|\theta_{\beta}^{\prime}(s)\right|<\cosh \theta_{\beta}(s), \quad \text { when } \cosh \theta_{\beta}(s) \leqq \sqrt{2}, \\
\left|\theta_{\beta}^{\prime}(s)\right|<2\left|\tanh \theta_{\beta}(s)\right|, \quad \text { when } \sqrt{2}<\cosh \theta_{\beta}(s) .
\end{array}\right.
\end{gathered}
$$

The components $\tilde{a}_{\imath \jmath}$, at the point $\tilde{\phi}_{\beta}\left(s, t_{2}, \ldots, t_{n}\right)$, of the shape operator of the isometric immersion $\tilde{f}$, are given by

$$
\left\{\begin{array}{l}
\tilde{a}_{11}=-r_{\beta}(s) T^{2}\left(P_{\beta} Q_{\beta}\right)^{-1} \cosh ^{2} \theta_{\beta}(s), \\
\tilde{a}_{1 \jmath}=-r_{\beta}(s) T Q_{\beta}^{-1} \cosh \theta_{\beta}(s) \sinh \theta_{\beta}(s) b_{2 j \beta}(s), \\
\tilde{a}_{i 1}=-\tilde{a}_{11} R_{\imath \beta}\left(T P_{\beta}\right)^{-1} \tanh \theta_{\beta}(s), \\
\tilde{a}_{\imath \jmath}=-\tilde{a}_{1 j} R_{\imath \beta}\left(T P_{\beta}\right)^{-1} \tanh \theta_{\beta}(s),
\end{array}\right.
$$

$i, j=2, \ldots, n$, where $P_{\beta}=T^{2}+t_{2}^{2} \sinh ^{2} \theta_{\beta}(s), Q_{\beta}=T \cosh \theta_{\beta}(s)+t_{2} \theta_{\beta}^{\prime}(s)+\sigma_{\beta}$ $\sinh \theta_{\beta}(s), \sigma_{\beta}=\sum_{j=3}^{n} t_{j} k_{j \beta}(s)$ and $R_{i \beta}=\left(T^{2}-t_{2}^{2}\right) b_{2 i \beta}(s)-\sum_{j=3}^{n} t_{2} t_{j} b_{j i \beta}(s) \cosh \theta_{\beta}(s)$. The $C^{\infty}$ functions $r_{\beta}(s), \theta_{\beta}(s), k_{3 \beta}(s), \ldots, k_{n \beta}(s)$ defined in $\left(a_{\beta}, b_{\beta}\right)$ satisfy the conditions that as $s$ approaches the end points of $\left(a_{\beta}, b_{\beta}\right)$,

$$
X_{1 \beta}^{l_{1}} X_{2 \beta}^{l_{2}} \cdots X_{n \beta}^{l_{n}}\left(\tilde{a}_{\imath \jmath}\right) \longrightarrow 0
$$

for all nonnegative integers $l_{1}, l_{2}, \ldots, l_{n}$. Here the vector fields $X_{\imath \beta}, i=1, \ldots, n$, in the product space $\left(a_{\beta}, b_{\beta}\right) \times R^{n-1}$ are given by

$$
\left\{\begin{array}{l}
X_{1 \beta}=\frac{T \cosh \theta_{\beta}(s)}{Q_{\beta}} X_{\beta}, \\
X_{\imath \beta}=-\frac{R_{\imath \beta} \sinh \theta_{\beta}(s)}{P_{\beta} Q_{\beta}} X_{\beta}+Y_{\imath \beta}, \quad i=2, \ldots, n .
\end{array}\right.
$$

Also $X_{\beta}$ and $Y_{\imath \beta}$ are the vector fields in $\left(a_{\beta}, b_{\beta}\right) \times R^{n-1}$ given by

$$
\left\{\begin{array}{l}
X_{\beta}=-\frac{\partial}{\partial s}+\frac{-t_{2} \sinh \theta_{\beta}(s) \theta_{\beta}^{\prime}(s)+\sigma_{\beta}}{\cosh \theta_{\beta}(s)} \frac{\partial}{\partial t_{2}}-\sum_{j=3}^{n} t_{2} k_{j \beta}(s) \cosh \theta_{\beta}(s) \frac{\partial}{\partial t_{j}}, \\
Y_{\imath \beta}=\frac{b_{2 i \beta}(s)}{\cosh \theta_{\beta}(s)} \frac{\partial}{\partial t_{2}}+\sum_{j=3}^{n} b_{j i \beta}(s) \frac{\partial}{\partial t_{j}}, \quad i=2, \ldots, n .
\end{array}\right.
$$

For each pair of parametrizations $\tilde{\phi}_{\alpha}$ as in (4.1) and $\tilde{\phi}_{\beta}$ as in (4.4), we have one of the following inequality: 


$$
\left\{\begin{array}{l}
\operatorname{const}(\alpha, \beta)<a_{\alpha}-b_{\beta}, \quad \text { or, } \\
\operatorname{const}(\beta, \alpha)<a_{\beta}-b_{\alpha},
\end{array}\right.
$$

where const $(\alpha, \beta)$ is a positive constant depending on the vectors $\tilde{e}_{3 \alpha}, \ldots, \tilde{e}_{n \alpha}$, and the functions $k_{3 \beta}(s), \ldots, k_{n \beta}(s)$ and $\theta_{\beta}(s)$ defined in $\left(a_{\beta}, b_{\beta}\right)$. In fact, if $a_{\beta}-b_{\alpha}<\pi / 2-\tan ^{-1} \rho(s), \forall s \in\left(a_{\beta}, b_{\beta}\right)$, where $\rho(s)=\lambda(s)\left(1+\mu(s)^{2} \sinh ^{2} \theta_{\beta}^{2}(s)\right)^{1 / 2}$, we can find $2 n$ real numbers $s \in\left(a_{\alpha}, b_{\alpha}\right), s^{\prime} \in\left(a_{\beta}, b_{\beta}\right)$, and $t_{2}, \ldots, t_{n}, t_{2}^{\prime}, \ldots, t_{n}^{\prime}$ such that

$$
\tilde{\phi}_{\alpha}\left(s, t_{2}, \ldots, t_{n}\right)=\tilde{\psi}_{\beta}\left(s^{\prime}, t_{2}^{\prime}, \ldots, t_{n}^{\prime}\right) .
$$

Operating a rotation of the Euclidean space $R^{n-1}$ on $e_{2 \beta}\left(s^{\prime}\right), \ldots, e_{n \beta}\left(s^{\prime}\right)$, if necessary, we may assume here that for each pair of real numbers $s \in\left(a_{\alpha}, b_{\alpha}\right), s^{\prime} \in$ $\left(a_{\beta}, b_{\beta}\right), e_{\imath \alpha}(s)=e_{\imath \beta}\left(s^{\prime}\right), i=4, \ldots, n$ and that

$$
\left\{\begin{array}{l}
e_{2 \alpha}=\lambda\left(s^{\prime}\right) e_{2 \beta}\left(s^{\prime}\right)+\mu\left(s^{\prime}\right) e_{3 \beta}\left(s^{\prime}\right), \\
e_{3 \alpha}= \pm\left\{-\mu\left(s^{\prime}\right) e_{2 \beta}\left(s^{\prime}\right)+\lambda\left(s^{\prime}\right) e_{3 \beta}\left(s^{\prime}\right)\right\},
\end{array}\right.
$$

for some suitable functions $\lambda\left(s^{\prime}\right)$ and $\mu\left(s^{\prime}\right)$.

Hence we have the following.

THEOREM 4.1. For a given isometric immersion $\tilde{f}$ of $\widetilde{H}_{1}^{n}$ into $\widetilde{H}_{1}^{n+1}$, there are a family of countable $r_{\alpha}(s)$ of real-valued functions defined in $\left(a_{\alpha}, b_{\alpha}\right)$ satisfying (4.3) such that the components $\tilde{a}_{\imath}$ of $\tilde{f}$, at $\phi_{\alpha}\left(s, t_{2}, \ldots, t_{n}\right)$, are given by (4.2), and a family of countable $n$-tuples $\left\{r_{\beta}(s), \theta_{\beta}(s), k_{3 \beta}(s), \ldots, k_{n \beta}(s)\right\}$ of real-valued functions defined in $\left(a_{\beta}, b_{\beta}\right)$ satisfying (4.5)-(4.8) and (4.10) such that the components $\tilde{a}_{\imath}$ of $\tilde{f}$, at $\phi_{\beta}\left(s, t_{2}, \ldots, t_{n}\right)$, are given by (4.9).

Conversely, suppose that there are given a family of countable $r_{\alpha}(s)$ of realvalued functions defined in $\left(a_{\alpha}, b_{\alpha}\right)$ satisfying (4.3) and a family of countable ntuples $\left\{r_{\beta}(s), \theta_{\beta}(s), k_{3 \beta}(s), \ldots, k_{n \beta}(s)\right\}$ of real-valued functions defined in $\left(a_{\beta}, b_{\beta}\right)$ satisfying (4.5)-(4.8) and (4.10). Assume further that (4.12) holds among $a_{\alpha}$ 's, $a_{\beta}$ 's, $\beta_{\alpha}$ 's and $b_{\beta}$ 's. Then, there is an isometric immersion $\tilde{f}$ of $\tilde{H}_{1}^{n}$ into $\widetilde{H}_{1}^{n+1}$ such that the components $\dot{a}_{\imath}$ of $\tilde{f}$ are given by (4.2) and (4.9).

Proof. The first half has already been shown above.

To obtain the second half, we only need to trace backward the argument for the first half.

\section{REFERENCES}

[1] AвE, E., Isometric immersions of $H_{1}^{n}$ into $H_{1}^{n+1}$, Pacific J. Math., 173 (1996), $1-27$.

[2] ABE, K. AND HAAS, A., Isometric immersions of $H^{n}$ into $H^{n+1}$, Differential Geometry: Riemannian Geometry, Proc. Sympos. Pure Math., 54, Part. 3, 1993, 23-30. 
[3] ABE, K. AND MAGID, M., Relative nullity foliations and indefinite isometric immersions, Pacific J. Math., 124 (1986), 1-20.

[4] Abe, K., MoRi, H. AND TAKahashi, H., A parametrization of isometric immersions between hyperbolic spaces, Geom. Dedicata, 65 (1997), 31-46.

[5] DAJCzER, M. ET AL., Submanifolds and Isometric Immersions, Math. Lecture Ser., 13, Publish or Perish, Houston, Texas, 1990.

[6] Graves, L.K., Codimension one isometric immersions between Lorentz spaces, Trans. Amer. Math. Soc., 252 (1979), 367-392.

[7] FeruS, D., Isometric immersions between hyperbolic spaces, Math. Ann., 205 (1973), 193-200.

[8] Helgason, S., Differential Geometry and Symmetric Spaces, Academic Press, New York, 1962.

[9] NomizU, K., Isometric immersions of the hyperbolic plane into the hyperbolic space, Math. Ann., 205 (1973), 181-192.

[10] O'Neill, B., Immersions of manifolds of non-positive curvature, Proc. Amer. Math. Soc., 11 (1960), 132-134.

[11] O'Neill, B. AND STIEL, E., Isometric immersions of constant curvature manifolds, Michigan Math. J., 10 (1963), 335-339.

[12] Wolf, J.A., Spaces of constant curvature, 5th ed., Publish or Perish, Houston, Tex., 1984.

\author{
DEPARTMENT OF MATHEMATICS \\ UNIVERSITY OF CONNECTICUT \\ U-9, MSB 111 \\ 196 AUdITORIUM ROAD \\ STORRS, CONNECTICUT 06269 \\ USA \\ e-mail : abe@math.uconn.edu \\ Departmen'T OR Mathematics \\ JOETSU UNIVERSITY OF EDUCATION \\ Joetsu, Nilgata Pref. 943 \\ JAPAN \\ e-mail : mori@juen.ac.jp
}

